Research Article

\title{
Prevalence and Factors Associated with Sexually Transmitted Infections among Jimma University Students, Southwest Ethiopia
}

\author{
Rahel Tamrat, Tesfaye Kasa, Zewdineh Sahilemariam, and Mulatu Gashaw iD \\ School of Medical Laboratory Science, Jimma University, Jimma, Ethiopia \\ Correspondence should be addressed to Mulatu Gashaw; mulatugashaw@gmail.com
}

Received 5 June 2020; Revised 30 September 2020; Accepted 14 October 2020; Published 10 November 2020

Academic Editor: Faham Khamesipour

Copyright (c) 2020 Rahel Tamrat et al. This is an open access article distributed under the Creative Commons Attribution License, which permits unrestricted use, distribution, and reproduction in any medium, provided the original work is properly cited.

Background. Globally, one-third of new sexually transmitted infection (STI) cases occur in people under 25 years of age every year. University students are in the youth age category and are exposed to risky sexual behaviors such as unprotected sexual intercourse leading to STIs. Therefore, this study was aimed to assess the prevalence and associated factors of STI among Jimma University students. Method. A health facility-based cross-sectional study was conducted at Jimma University students' clinic from April 2017 to October 2017 among students with STI syndromes. Urethral, endocervical, and vaginal discharge swabs were collected by attending nurses. Standard protocol was followed to detect the etiologies of STI. Data were entered and analyzed using SPSS Version 20. Results. The overall prevalence of STIs among clinically suspected university students was $14.3 \%$. The predominant causes of STI were Neisseria gonorrhoeae (7.4\%) followed by T. vaginalis (4.8\%) and T. pallidum (3.7\%). Having had sex after taking alcohol was significantly associated with STIs $(P \leq 0.000)$. All $N$. gonorrhoeae isolates were found to be resistant to penicillin and tetracycline. Conclusion. In this study, the prevalence of STI was relatively high. The isolated N. gonorrhoeae was resistant to commonly prescribed antibiotics. Therefore, evidence-based treatment and proper reproductive health education for youth are highly recommended.

\section{Introduction}

Sexually transmitted infections (STIs) are mainly transmitted from person-to-person through sexual contact [1]. There are several counts of sexually transmissible etiologies from members of bacteria, viruses, and parasites. These are responsible for multiple sexually transmissible diseases such as gonorrhea, chlamydial infection, syphilis, trichomoniasis, chancroid, genital herpes, human immunodeficiency virus (HIV) infection, and hepatitis B infection. Some of the above etiologies can also be transmitted vertically from mother to child during pregnancy as well as through blood products [2]. From all recognized STIs, four of them, namely, syphilis, gonorrhea, chlamydia, and trichomoniasis, have been found to be curable [3].

Sexually transmitted infections are a major cause of acute illness, infertility, long-term disability, and death with serious medical and psychological consequences of millions of individuals [2]. Both ulcerative and inflammatory curable STIs increase the risk of acquiring and transmitting HIV by up to two- to threefold [4]. Globally, one-third of over 340 million new STI cases occur per year in people under the age of 25 years. In developing countries particularly in subSaharan Africa, the burden of STI is very high with 108 million STIs occurring every day. It is estimated that 80 to $90 \%$ of the global burden of STIs occur in those low-income countries, where there is limited or no access to diagnostic facilities and poor awareness [5].

About 35\% of Ethiopian population comprised young people between 15 and 24 years of age, and this largest category is highly vulnerable to STIs $[6,7]$. In a STI surveillance study conducted in 8 health facilities in various regions of Ethiopia, younger people in the age group of $20-34$ years were among highly affected ones (68.2\%) [8]. Most university students are in the youth age category and are exposed to risky sexual behaviors which lead to acquiring STIs [9]. According to a cross-sectional study conducted in 
one of the universities in Ethiopia, the prevalence of selfreported STIs was $19.5 \%$ among university students [10].

Even though few studies on STIs were done in Ethiopia, there are no adequate data about the prevalence and associated risk factors of $N$. gonorrhoeae, syphilis, and $T$. vaginalis infection and antimicrobial susceptibility patterns of $N$. gonorrhoeae isolates among university students in Ethiopia. Therefore, this study was aimed to determine the prevalence of STIs specifically N. gonorrhoeae, T. pallidum, and T. vaginalis infection and their associated risk factors and antimicrobial susceptibility patterns of $N$. gonorrhoeae among Jimma University students.

\section{Methods}

2.1. Study Design and Period. A health facility-based crosssectional study was conducted from April 1, 2017, to October 5, 2017, at Jimma University students' clinic. Jimma University is located in Ethiopia and $352 \mathrm{~km}$ far from the capital. It serves for more than 30,000 students in different programs.

2.2. Sample Size. All students who came to student clinic with STI syndromes in the study period were included except students who took antibiotics or antiprotozoan treatment two weeks prior to enrollment in the study. Therefore, a total of 189 university students participated in the study.

\subsection{Data Collection}

2.3.1. Sociodemographic and Sexual Behavior. A pretested structured self-administered questionnaire was used to collect data on sociodemographic and risky sexual behaviors.

\subsection{Laboratory Data}

2.4.1. Specimen Collection and Processing. Two cervical swabs and one vaginal swab were taken from female students, while three urethral swabs were taken from male students by attending nurses. The first cervical or urethral swab was inserted aseptically in the Amies transport medium to transport to Jimma Medical Center Microbiology Laboratory where isolation, identification, and antimicrobial susceptibility testing were done for $N$. gonorrhoeae. The second cervical or urethral swabs were smeared and gram stained for microscopical examination. The last vaginal swab or urethral swab was added to a tube containing sterile $0.5 \mathrm{ml}$ physiologic saline for wet-mount preparation to identify $T$. vaginalis. Three to five milliliters of venous blood were collected into anticoagulant-free sterile tubes for serological screening of $T$. pallidum infection using One Rapid immunochromatographic test kit (Inc., China).

The first cervical or urethral swabs transported with Amies transport medium were inoculated on the Modified Thayer Martin medium (Oxoid, England) and incubated at $35-37^{\circ} \mathrm{C}$ in the presence of $5 \% \mathrm{CO}_{2}$ for $24-72$ hours and examined for the presence growth every 24 hours of incubation. If growth was observed, single colony was taken and subcultured on a GC-chocolate agar supplemented with 1\% Vitox (Oxoid, England) and incubated in similar fashion. To identify the pathogen, gram staining and standard biochemical tests (oxidase, superoxol, and carbohydrate utilization tests) and Analytical Profile Index for identification of Neisseria and Haemophilus (API NH) kit strips (bioMerieux, France) were used [11].

2.4.2. Antimicrobial Susceptibility Testing. From the GCchocolate agar culture medium, 3-5 isolated colonies of bacteria were transferred and added to a tube containing $2.5 \mathrm{ml}$ sterile normal saline until the turbidity of the suspension becomes comparable to $0.5 \mathrm{McFarland}$ standard. The bacterial suspensions were swabbed evenly to distribute the bacteria over the entire surface of GC-chocolate agar with $1 \%$ Vitox supplement using a sterile swab. The antimicrobial susceptibility patterns of isolates were assessed to the following antimicrobial agents: penicillin (P10 IU), tetracycline (TE, $30 \mu \mathrm{g}$ ), ciprofloxacin (CIP, $5 \mu \mathrm{g}$ ), ceftriaxone (CRO, $30 \mu \mathrm{g}$ ), cefixime (CFM, $5 \mu \mathrm{g}$ ), and cefoxitin (FOX, $30 \mu \mathrm{g}$ ) (all from Oxoid, England) according to CLSI guidelines [12].

2.5. Quality Control. Culture media were tested for sterility before being used to process the clinical specimen and recovery rate using standard reference strain of $N$. gonorrhoeae ATCC 49226 to isolate the intended microbes and to perform antimicrobial susceptibility testing of gonococcal isolates according to CLSI guidelines [12].

2.6. Data Analysis. Data were entered into Epi data software version 4.0 and exported to SPSS version 20.0 for statistical analyses. Descriptive statistics and chi-square tests were carried out. Bivariate and multivariate logistic regressions were employed to determine the risk factors of STIs, and a $P$ value $\leq 0.05$ was taken as statistically significant.

2.7. Ethical Consideration. Ethical clearance was obtained from Jimma University, Institutional Review Board. Support letter was obtained from school of medical laboratory sciences and student service dean of Jimma University. Written informed consent was obtained from each participant. All the laboratory test results were given for treating physicians for beneficiary of the patients.

\section{Results}

A total of 189 university students suspected for sexual transmitted infections were included in the study with $100 \%$ response rate. Fifty-eight (30.7\%) students were males, and three-fourth were twenty or greater than twenty years of age. The distribution of the students across the university institute or college appeared fairly uniform between $22.2 \%$ and $27.5 \%$. Forty-nine $(29.5 \%)$ university students were first year, whereas the remaining were second year and above. 
TABLE 1: Proportion of sexually transmitted infections among Jimma University students $(n=27)$.

\begin{tabular}{|c|c|c|c|c|}
\hline \multicolumn{2}{|c|}{ Sociodemographic characteristics } & \multicolumn{3}{|c|}{ Sexually transmitted infections } \\
\hline & & N. gonorrhoeae & T. vaginalis & T. pallidum \\
\hline \multirow{3}{*}{ Gender } & Male & $9(15.5)$ & $0(0)$ & $3(5.2)$ \\
\hline & Female & $5(3.8)$ & $9(6.9)$ & $4(3.1)$ \\
\hline & Health institute & $2(4.8)$ & $2(4.8)$ & $2(4.8)$ \\
\hline \multirow{4}{*}{ Faculty/college } & Social sciences and law & $3(5.9)$ & $5(9.8)$ & $3(5.9)$ \\
\hline & Natural science & $4(7.7)$ & $2(3.8)$ & $1(1.9)$ \\
\hline & Business and economics & $5(11.4)$ & $0(0)$ & $1(2.3)$ \\
\hline & Year I & $3(6.1)$ & $5(10.2)$ & $3(6.1)$ \\
\hline \multirow{2}{*}{ Academic year } & Year II & $4(6.6)$ & $2(3.3)$ & $3(4.9)$ \\
\hline & Year III and above & $7(8.9)$ & $2(2.5)$ & $1(1.3)$ \\
\hline \multirow{2}{*}{ Age in years } & $<20$ years & $2(4.4)$ & $4(8.7)$ & $2(4.3)$ \\
\hline & $\geq 20$ years & $12(8.4)$ & $5(3.5)$ & $5(3.5)$ \\
\hline
\end{tabular}

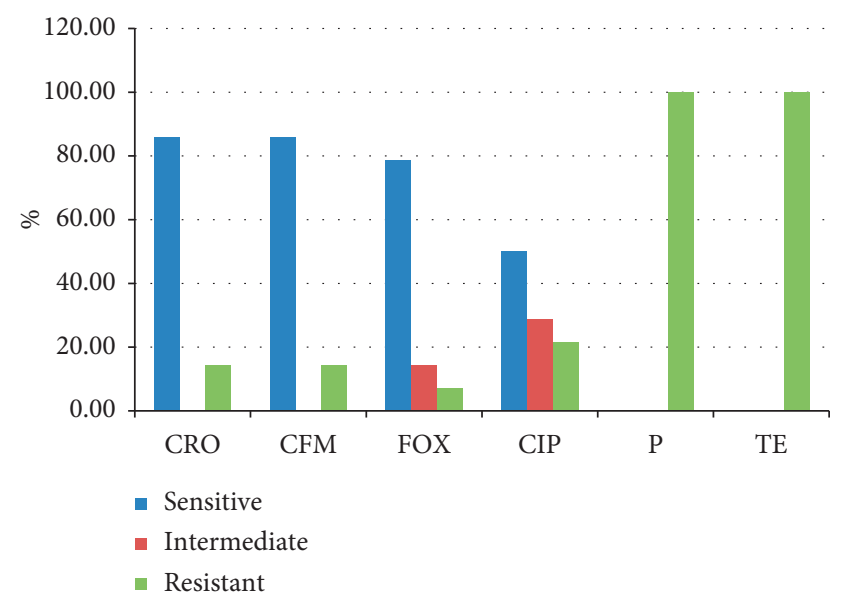

FIGURE 1: Antimicrobial susceptibility patterns of N. gonorrhoeae isolated from suspected university students for STI. P: penicillin; TE: tetracycline; CIP: ciprofloxacin; CRO: ceftriaxone; FOX: cefoxitin; CFM: cefixime.

From the total of 189 students suspected for STIs, 27 $(14.3 \%)$ of them were infected by one or more of the three etiologies. The prevalence of $N$. gonorrhoeae, T. pallidum, and T. vaginalis was $7.4 \%$ (14/189), 3.7\% (7/189), and $4.8 \%$ (9/189), respectively. Two patients were coinfected with $N$. gonorrhoeae and syphilis and another one patient with $N$. gonorrhoeae and T. vaginalis. Of the 14 patients who were confirmed to have gonococcal infection, nine $(15.5 \%)$ were males and five $(3.8 \%)$ were females. Of all study participants infected with $T$. vaginalis, nine $(6.9 \%)$ were females. There was no male patient positive for $T$. vaginalis, and $3(5.2 \%)$ males and $4(3.1 \%)$ females were infected with $T$. pallidum (Table 1).

In this study, the susceptibility pattern of gonococcus was done against six antimicrobial agents and showed that $85.7 \%$ of the isolates were susceptible to ceftriaxone and cefixime. On the contrary, $78.6 \%$ and $50 \%$ of the isolates were susceptible to cefoxitin and ciprofloxacin, respectively. Intermediate resistance was observed in $28.6 \%$ and $14.3 \%$ of the isolates against ciprofloxacin and cefoxitin, respectively. All gonococcus isolates were found to be resistant to penicillin and tetracycline (Figure 1).
To assess the risk factors for sexually transmitted infection, both bivariate and multivariate logistics regression analyses were done. From sexual behavior and substance use, having more than one sexual partner, having had sex after taking alcohol, and khat chewing were the candidate variables for multivariate logistic regression analysis. Finally, in the multivariate logistic regression analysis, only patients who had sex after using alcohol were independently associated with sexually transmitted infections $(P \leq 0.000)$ (Table 2).

\section{Discussion}

This study provides the prevalence and associated risk factors for STIs (N. gonorrhoeae, T. vaginalis, and T. pallidum) among Jimma University students suspected for sexually transmitted infections. The overall prevalence of STIs was $14.3 \%$. In the present study, the cause of most prevalent sexually transmitted infection was $N$. gonorrhoeae (7.4\%), which is higher than the studies reported from Hawassa (5.1\%) [13], Port Harcourt Rivers state (5\%) [14], and Abuja University (2.9\%) [15] and lower than the studies reported from Gambella (11.3\%), [16], Jimma (17.2\%) [17], Gondar (20.8\%) [18], Southern Mozambique (14\%) [19], and Uganda (59\%) [20]. The variation in prevalence rate might be because of variation in study population.

In this study, the prevalence of gonococcal infection was significantly higher in males $(15.5 \%)$ than females $(3.8 \%)$. This finding is comparable with the finding from Gambella in Ethiopia [16]. This is likely because gonococcal infections are more symptomatic in males than females that may take them for health-seeking decision.

In the present study, the second prevalent STI etiology was found to be T. vaginalis (4.8\%), which is similar with the finding reported from Abuja University (4.7\%) [15] and lower than the studies reported from Babcock University (12.5\%) [21] and Brazil (9\%) [22]. On the contrary, the prevalence of this study is higher than the study conducted in Nairobi (0.4\%) [23]. The observed difference in the rate of infection could be due to variation in the age distribution and personal hygiene practice. All students with $T$. vaginalis were females, and similar finding was reported from Abuja 
TABLE 2: Risk factors for sexually transmitted infections among university students $(n=189)$.

\begin{tabular}{|c|c|c|c|c|c|c|c|}
\hline \multirow{2}{*}{ Variables } & & \multicolumn{2}{|c|}{ STI } & \multicolumn{2}{|l|}{ COR } & \multicolumn{2}{|l|}{ AOR } \\
\hline & & Positive & Negative & $95 \% \mathrm{CI}$ & $P$ value & $95 \% \mathrm{CI}$ & $P$ value \\
\hline \multirow{2}{*}{ Sexual partner } & One & $8(6.00)$ & $125(94.0)$ & $1.00^{*}$ & & & \\
\hline & More than one & $19(33.9)$ & $37(66.1)$ & $8.02(3.5-19.81)$ & 0.000 & $2.09(0.62-7.10)$ & 0.223 \\
\hline \multirow{2}{*}{ Condom usage } & Yes & $13(12.3)$ & $93(87.7)$ & $1.00^{*}$ & & & \\
\hline & No & $14(16.9)$ & $69(83.1)$ & $1.45(0.64-3.29)$ & 0.371 & & \\
\hline \multirow{2}{*}{ Sex after taking alcohol } & Yes & $20(50.0)$ & $20(50.0)$ & $20.29(7.62-54.04)$ & 0.000 & $18.79(5.00-70.7)$ & 0.000 \\
\hline & No & $7(4.7)$ & $142(95.3)$ & $1.00^{*}$ & & & \\
\hline \multirow{2}{*}{ Condom use after taking alcohol } & Yes & $5(25.0)$ & $15(75.0)$ & $1.00^{*}$ & & & \\
\hline & No & $7(35.0)$ & $13(65.0)$ & $1.62(0.41-6.34)$ & 0.492 & & \\
\hline \multirow{2}{*}{ Chewing khat } & Yes & $6(22.2)$ & $21(77.8)$ & $1.92(0.69-5.30)$ & 0.209 & $1.5(0.41-5.59)$ & 0.539 \\
\hline & No & $21(13.0)$ & $141(87.0)$ & $1.00^{*}$ & & & \\
\hline
\end{tabular}

$*$ Reference value.

University [15] and Gondar [18]. Female patients are more symptomatic to this protozoan vaginosis than males.

On the contrary, the prevalence of T. pallidum infection was $3.7 \%$ which is higher than the one reported from Addis Ababa (0\%) [24], Jigjiga (0.1\%), [25] and Abuja 2.4\% [15]. The target population in Addis Ababa study was women who attended antenatal follow-up and healthy blood donors in the Jigjiga study. On the contrary, the prevalence of T. pallidum in the current study is lower compared with the study in Beijing (7\%) [26], Zambia (6.5\%) [27], and Nigeria $(15.4 \%)$ [28]. The variation in the prevalence rate of infection may be due to differences in target population.

In the present study, complete resistance to penicillin and tetracycline was reported which was similar to reports in Gambella [16] and India [29]. Such resistant features may be due to beta-lactamase producing $N$. gonorrhoeae strains. The bacteria also showed about $20 \%$ resistance to ciprofloxacin which is in line with the reported results from Hawassa (28\%) [13], Gambella (18\%) [16], and Uganda (23\%) [20]. This might be because of the intensive use of ciprofloxacin antimicrobial agent as part of the guideline in syndromic management of gonococcus without evidence. Cefoxitin was found to be effective in $78.6 \%$ of the isolates although it is relatively lesser than that reported from Hawassa (82\%) [13] and Gambella (100\%) [16].

In this finding, $N$. gonorrhoeae showed $14.3 \%$ resistance to ceftriaxone, which is lower than the studies reported from Bahir Dar (27.8\%) [30], Southern Ethiopia (33.3\%) [31], and India (40\%) [29] and total resistance in Iran [32]. Multidrug resistance was observed in all $N$. gonorrhoeae isolates. Currently, extensively drug-resistant $N$. gonorrhoeae strains have been reported in Japan [33], France [34], and Spain [35] that displayed a high level of resistance to cefixime and ceftriaxone. In our study, having had sex after taking alcohol was independently associated with STI $(P \leq 0.000)$. This might be due to alcohol consumption, having an instating effect that may make engaged in risky sexual behaviors.

\section{Conclusion}

In this study, the prevalence of STI due to N. gonorrhoeae, T. vaginalis, and T. pallidum was found to be high among university students. As a key factor, having had sex after taking alcohol showed significant association with STIs. $N$. gonorrhoeae has shown increased resistance to thirdgeneration cephalosporins like ceftriaxone and cefixime. Evidence-based treatment and continuous antimicrobial resistance surveillance are highly recommended. Proper education on reproductive health for university students has invaluable importance to prevent sexually transmitted infections.

\section{Data Availability}

The data used to support the findings of this study are included in the manuscript and are available from the corresponding author on reasonable request.

\section{Disclosure}

All the data used to support the findings of this study are included in the manuscript.

\section{Conflicts of Interest}

The authors declare that there are no conflicts of interest.

\section{Authors' Contributions}

RT, TK, ZS, and MG participated in design, write-up, analysis, and interpretation of data. RT and MG participated in data collection, laboratory work, and prepared the manuscript. All the authors reviewed and approved the final version. All authors read and approved the final manuscript.

\section{Acknowledgments}

We are grateful to Jimma University for the financial support to conduct this research. We thank the study participants for their willingness to be part of this study.

\section{References}

[1] K. A. Workowski and G. A. Bolan, "Sexually transmitted diseases treatment guidelines," MMWR Morbidity and Mortality Weekly Report, vol. 64, pp. 1-137, 2015. 
[2] WHO, Global Incidence and Prevalence of Selected Curable Sexually Transmitted Infections-2008, World Health Organization, Geneva, Switzerland, 2012.

[3] WHO, 10 Facts on Sexually Transmitted Infections, World Health Organization, Geneva, Switzerland, 2013.

[4] R. Hayes, D. Watson-Jones, C. Celum, W. Jvd, and J. Wasserheit, "Treatmentof sexually transmitted infections for HIV prevention: end of the road or newbeginning," Acquired Immunodeficiency Syndrome, vol. 24, no. Suppl 4, pp. S15-S26, 2010.

[5] WHO and Department of Reproductive Health and Research, Global Strategy for the Perevention and Control of Sexually Transmitted Infections: 2006-2015, World Health Organization, Geneva, Switzerland, 2007.

[6] United States Agency for International Development and Path Finder International, Bringing Youth Friendly Services to Scale in Ethiopia, pp. 1-8, USAID, Washington, DC, USA, 2012.

[7] Y. Berhane, Y. Mekonnen, E. Seyoum, L. Gelmon, and D. Wilson, HIV/AIDS in Ethiopia an Epidemiological Synthesis World Bank Global HIV/AIDS Program, UNAIDS, Geneva, Switzerland, 2008.

[8] Federal Democratic Republic of Ethiopia Ministry of Health, National Guidelines for the Management of Sexually Transmitted Infections Using Syndromic Approach, Federal Democratic Republic of Ethiopia Ministry of Health, Addis Ababa, Ethiopia, 2015, https://www.medbox.org/document/ national-guidelines-for-the-management-of-sexuallytransmitted-infections-using-syndromic-approach-ethiopia.

[9] G. Tura, F. Alemseged, and S. Dejene, "Risky sexual behaviour and predisposing factors among students of Jimma University," Ethiopian Journal of Health Sciences, vol. 22, no. 3, pp. 170-180, 2012.

[10] B. Yohannes, T. Gelibo, and M. Tarekegn, "Prevalence and associated factors of sexually transmitted infections among students of wolaita sodo university, southern Ethiopia," International Journal of Scientific \& Technology Research, vol. 2, no. 2, 2013.

[11] M. Cheesbrough, District Laboratory Practice in Tropical Countries Part 2pp. 90-97, Cambridge University Press, Cambridge, UK, 2nd edition, 2006.

[12] CLSI, Performance Standards for Antimicrobial Susceptibility Testing CLSI Supplement M100S, Clinical and Laboratory Standards Institute, Wayne, PA, USA, 26th edition, 2016.

[13] M. Hailemariam, T. Abebe, A. Mihret, and L. Tariku, "Prevalence of Neisseria gonorrhea and their antimicrobial susceptibility patterns among symptomatic women attending gynecology outpatient department in Hawassa referral hospital, Hawassa," Ethiopian Journal of Health Sciences, vol. 23, no. 1, pp. 10-18, 2013.

[14] T. W. Kennedy and O.L. Ibinabo, "Prevalence of Neisseria gonorhoea among under graduate female students of university of Port harcpurt using strand displacement and amplification (sda) technique," Journal of Dental and Medical Sciences, vol. 7, no. 4, pp. 76-79, 2013.

[15] Y. Thairu, H. A. Shehu, and N. Egenti, "Profile of sexually transmitted infections (STIs) among students of tertiary educational institution in Abuja, Nigeria," IOSR Journal of Dental and Medical Sciences, vol. 14, no. 11, pp. 71-74, 2015.

[16] S. Ali, T. Sewunet, Z. Sahlemariam, and G. Kibru, "Neisseria gonorrhoeae among suspects of sexually transmitted infection in Gambella hospital, Ethiopia: risk factors and drug resistance," BMC Research Notes, vol. 9, no. 439, 2016.
[17] M. Shimelis, A. Mossie, and S. Fekadu, "Substance use and sexually transmitted infections among anti-retroviral treatment attendees in Jimma university specialized hospital, Jimma, Ethiopia," Ethiopian Journal of Health Sciences, vol. 22, no. 3, pp. 181-188, 2012.

[18] A. G. Rozina, M. A. Beyene, A. B. Abate, E. S. Mengistu, and G. Y. Addisu, "Prevalence of selected sexually transmitted infection (STI) and associated factors among symptomatic patients attending gondar town hospitals and health centers," Ethiopian Journal of Health Sciences, vol. 27, no. 6, p. 589, 2017.

[19] C. Menéndez, X. Castellsagué, M. Renom et al., "Prevalence and risk factors of sexually transmitted infections and cervical neoplasia in women from a rural area of southern Mozambique," Infectious Diseases in Obstetrics and Gynecology, vol. 11, pp. 1-9, 2010.

[20] P. A. Florence, F. Otim, F. Okongo, M. Ogwang, and D. Greco, "The prevalence and antibiotics susceptibility pattern of Neisseria gonorrhoeae in patients attending OPD clinics at St. Mary's Hospital Lacor Uganda," Journal of Preventive Medicine and Hygiene, vol. 53, pp. 186-189, 2012.

[21] H. S. John, S. S. Enitan, O. Adeyemi, G. E. Itodo, G. O. Otuneme, and A. O. Olayanju, "The prevalence of trichomonas vaginalis infection and associated risk factors among undergraduate female students of Babcock university, Ilishan-Remo, Ogun state, Nigeria," International STD Research \& Reviews, vol. 6, no. 1, pp. 1-13, 2017.

[22] C. L. Ambrozio, A. S. Nagel, S. Jeske, G. C. M. Bragança, S. Borsuk, and M. M. Villela, "Trichomonas vaginalis prevalence and risk factors for women in Southern Brazil," Revista do Instituto de Medicina Tropical de São Paulo, vol. 58, no. 61, 2016.

[23] A. N. Maina, J. Kimani, and O. Anzala, "Prevalence and risk factors of three curable sexually transmitted infections among women in Nairobi, Kenya," BMC Research Notes, vol. 9, p. 193, 2016.

[24] F. Kinfe, A. Ibrahim, and G. Ashebir, "Seroprevalence and risk factors of sexually transmitted infections (HIV, HBV and syphilis) among pregnant women provided health care services, Addis Ababa, Ethiopia," American Journal of Health Research, vol. 5, no. 5, pp. 154-161, 2017.

[25] Y. Mohammed and A. Bekele, "Seroprevalence of transfusion transmitted infection among blood donors at Jijiga blood bank, Eastern Ethiopia: retrospective 4 years study," BMC Research Notes, vol. 9, no. 129, 2016.

[26] J. Zheng, Z. Wu, E. Katharine, K. E. Poundstone, L. Pang, and K. Rou, "HIV, syphilis infection, and risky sexual behaviors among male university students who have sex with men in beijing, China: a cross-sectional study," AIDS Education and Prevention, vol. 24, no. 1, pp. 78-88, 2012.

[27] C. Machila, H. T. Nyirenda, and C. K. Mulele, "Prevalence and risk factors associated with syphilis at the copperbelt university riverside campus, kitwe Zambia," Asian Pacific Journal of Health Sciences, vol. 4, no. 3, pp. 59-65, 2017.

[28] E. A. Ophori, O. Atanunu, E. J. Johnny, and M. Adu, "Sero perevalance of syphilis in apparently healthy students from tertiary institution in Benin city, Nigeria," Japanese Journal of Infectious Diseases, vol. 63, pp. 437-439, 2010.

[29] R. Sangita, S. Arpita, P. Aarohi, and M. Summaiya, "Surveillance of gonococcal infections with assessment of their antibiotic susceptibility pattern," Advances in Infectious Diseases, vol. 6, no. 2, pp. 46-51, 2016.

[30] M. Tibebu, A. Shibabaw, G. Medhin, and A. Kassu, "Neisseria gonorrhoeae non-susceptible to cephalosporins and 
quinolones in Northwest Ethiopia," BMC Infectious Diseases, vol. 13, p. 415, 2013.

[31] A. Amsalu, Z. Geto, D. Asegu, and E. Setegn, "Antimicrobial resistance pattern of bacterial isolates from different clinical specimens in Southern Ethiopia: a three year retrospective study," African Journal of Microbiology Research, vol. 9, pp. 1-8, 2017.

[32] S. Afrasiabi, R. Moniri, M. Samimi, and S. G. Mousavi, "The frequency of Neisseria gonorrhoeae endocervical infection among female carrier and changing trends of antimicrobial susceptibility patterns in Kashan, Iran," Iranian Journal of Microbiology, vol. 6, no. 3, pp. 194-7, 2014.

[33] M. Ohnishi, D. Golparian, K. Shimuta et al., "Is Neisseria gonorrhoeae initiating a future era of untreatable gonorrhea?: detailed characterization of the first strain with high-level resistance to ceftriaxone," Antimicrobial Agents and Chemotherapy, vol. 55, no. 7, pp. 3538-3545, 2011.

[34] M. Unemo, D. Golparian, R. Nicholas, M. Ohnishi, A. Gallay, and P. Sednaoui, "High-level cefixime- and ceftriaxone-resistant Neisseria gonorrhoeae in France: novel penA mosaic allele in a successful international clone causes treatment failure," Antimicrobial Agents and Chemotherapy, vol. 56, no. 3, pp. 1273-1280, 2012.

[35] J. Camara, J. Serra, J. Ayats et al., "Molecular characterization of two high-level ceftriaxone-resistant Neisseria gonorrhoeae isolates detected in Catalonia, Spain," Journal of Antimicrobial Chemotherapy, vol. 67, no. 8, pp. 1858-1860, 2012. 